\title{
ВЛИЯНИЕ РОДСТВЕННОГО УХОДА НА ЗАНЯТОСТЬ, ЗДОРОВЬЕ И МАТЕРИАЛЬНОЕ ПОЛОЖЕНИЕ УХАЖИВАЮЩИХ
}

\author{
ЕЛЕНА ГРИШИНА, ЕЛЕНА ЦАЦУРА
}

\begin{abstract}
В России, как и в большинстве других стран, основная нагрузка по уходу за пожильми и инвалидами ложится на родственников. В статье на основе данных обследования «Комплексное наблюдение условий жсизи населения» Росстата за 2018 г. проанализировано влияние родственного ухода на положение ухаживаюших лии.. В частности, показано, что люди трудоспособного возраста, ухаживающие за родственниками, реже имеют работу; чаще работают неполный рабочий день или неполную рабочую неделю; чащзе имеют хронические заболевания и более низкую самооценку здоровья. Интенсивный уход (более 20 часов в неделю) усиливает негативное влияние на занятость и самооиенку здоровья. Среди лиц трудоспособного возраста, осуществляюших интенсивный родственный уход, выше доля бедных и крайне бедных и ниже доля лии со среднедушевым доходом выле двукратной величины прожиточного минимума. Это свидетельствует о необходимости создания комплексной системы мер, направленных на поддержку родственников, осуществляющих уход, включая меры по сохранению занятости и развитию гибких форм занятости на рынке труда, расширению охвата лиц, имеющих родственников и нуждающихся в уходе, программами временного и дневного ухода, по оказанию консультационной поддержки родственников и организачии очного $и$ дистанционного обучения родственников навыкам ухода. Усиление поддержки родственников, осуществляющих уход, позволит людям пожилого возраста и инвалидам, нуждающимся в уходе, как можно дольше оставаться дома в привычных условиях и получать при этом качественный уход.
\end{abstract}

Ключевые слова: родственный уход, долговременный уход, пожилые, лищуа осуществляющие уход.

\section{ВВЕДЕНИЕ}

По всему миру увеличивается доля населения в старших возрастах. В частности, по данным ОЭСР, в 1950 г. только 1\% всех людей были в возрасте старше 80 лет, к 2010 г. их было уже 4\%, к 2050 г. ОЭСР прогнозирует рост их доли до 10\% (Colombo et al. 2011). Это обостряет проблему ухода за пожилыми людьми. В России, как и в большинстве других стран, именно на родственников ложится основная нагрузка по уходу за пожилыми и людьми с ограниченными возможностями здоровья, что не может не сказываться тем или иным образом на их социально-экономическом положении.

ЕЛЕНА ЕвГЕНЬЕВНА ГРИШИНА (grishina@ranepa.ru), ИНСТИТУТ СОЦИАЛЬНОГО АНАЛИЗА И ПРОГНОЗИРОВАНИЯ РАНХИГС, РосСия.

ЕЛЕНА АЛЕКСЕЕВНА ЦАЦУРА (tsatsura-ea@ranepa.ru), ИНСТИТУТ СОЦИАЛЬНОГО АНАЛИЗА И ПРОГНОЗИРОВАНИЯ РАНХиГС, РОССИЯ.

РУКОПИСЬ ПОДГОТОВЛЕНА В РАМКАХ ВЫПОЛНЕНИЯ НАУЧНО-ИССЛЕДОВАТЕЛЬСКОЙ РАБОТЫ ГОСУДАРСТВЕННОГО ЗАДАНИЯ РАНХИГС

СТАТЬЯ ПОСТУПИЛА В РЕДАКЦИЮ В АПРЕЛЕ 2020 Г. 
В отечественной научной литературе тема влияния родственного ухода на положение ухаживающих не разработана, в то время как в мире целый пласт исследований, как количественных (например, Carmichael, Charles, Hulme 2010; van den Berg, Fiebig, Hall 2014; Niimi 2016), так и качественных (например, Lane, McKenna, Ryan 2003; Greenwood et al. 2019; Denham et al. 2019) посвящен тому, каким образом уход за престарелыми членами семьи или за людьми с ограниченными возможностями здоровья влияет на жизнь тех, кто ухаживает. Несмотря на то, что в большинстве работ говорится о негативном влиянии, есть и исследования, в которых уделяется внимание позитивным эффектам ухода для здоровья и благополучия ухаживающих (Brown, Brown 2014). Достаточно много статей посвящено проблеме двойной нагрузки, так называемого сэндвич-поколения, заботящегося одновременно о детях и о престарелых родителях (Attias-Donfut, Ogg, Wolf 2005; Grundy, Henretta 2006; Li, Song, Feldman 2009; McGarrigle, Kenny 2013).

Поддержка лиц, занятых родственным уходом (поддержка со стороны семьи и друзей, в большинстве случаев неоплачиваемая), крайне важна, учитывая, что для людей трудоспособного возраста основной проблемой является сложность совмещения оказываемой помощи и оплачиваемой работы. Им чаще всего приходится делать выбор в пользу сокращения рабочих часов или полного отказа от работы, что не только сказывается на их доходе, но и усложняет поиски работы в дальнейшем и уменьшает вероятность возврата на рынок труда. Помимо этого, такой вид долговременного ухода может привести к увеличению уровня стресса, что потенциально может привести к ухудшению физического и психического здоровья (Colombo et al. 2011).

В международных исследованиях, посвященных положению лиц, осуществляющих уход за своими родственниками и друзьями с ограниченными возможностями здоровья или в старших возрастах, главный вывод состоит в том, что в странах, где основным видом ухода за пожилыми и инвалидами является неформальный уход на дому, должны внедряться меры, в первую очередь призванные обеспечить сохранение трудового дохода лиц, предоставляющих уход, а также снижение финансовых, физических и психологических затрат на уход.

В исследованиях под долговременным неформальным уходом обычно понимается интенсивный уход, когда человек определенное количество часов в неделю ухаживает за тем, кто полностью или частично потерял возможность выполнять ежедневные действия, связанные с самообслуживанием (Activities of daily living, ADL), а также с возможностью самостоятельно проживать и выполнять повседневные дела (совершение покупок, заполнение документов, готовка и др.) (Instrumental activities of daily living, IADL). Концепция ADL и IADL (Katz et. al 1963) для определения возможностей выполнения повседневных действий для самообслуживания и самостоятельного существования широко используется во всем мире. Однако более четкое определение долговременного ухода дать затруднительно в связи с тем, что оно варьируется от страны к стране.

Один из самый важных аспектов - влияние ухода на уровень занятости. Исследования европейских, канадских и американских ученых показывают, что когда человек ухаживает за своим близким, уровень занятости снижается, (Carmichael, Charles 2003; Heitmueller 2007; Viitanen 2010; Latif 2013). 
При этом влияние сильнее в случае совместного проживания. Кроме того, сильно выражен гендерный аспект, женщинам особенно трудно сочетать оплачиваемую работу с осуществлением ухода. Межстрановое исследование также подтверждает, что влияние на занятость становится значимым при высокой интенсивности ухода (более 20 часов в неделю) и только в случае совместного проживания (Colombo et al. 2011).

Анализ, проведенный на базе данных Европейского обследования качества жизни (2011-2012), показал, что в ряде стран с наибольшим негативным влиянием ухода за родственниками на занятость сталкиваются люди в возрасте 18-44 года. Также подтверждается сильная гендерная составляющая, так как большинство ухаживающих - женщины (Rodrigues et al. 2013).

Многие люди продолжают работать, несмотря на то, что осуществляют уход более 20 часов в неделю. А представительницы «сэндвич-поколения» еще несут дополнительную нагрузку по уходу за детьми (Brodsky, Habib 2003).

По всем эффектам ситуация усугубляется с ростом объемов предоставляемой поддержки родственникам. Интенсивность ухода оказывает значимое влияние не только на участие на рынке труда, но и на уровень заработной платы (Carmichael, Charles 1998).

После преодоления порогового значения в 20 часов ухода в неделю снижается вероятность трудовой занятости ухаживающего, а также размер его часовой оплаты по сравнению с теми, кто не осуществляет уход. При этом, если на уход за близким человеком тратится менее 20 часов в неделю, то вероятность трудовой занятости у ухаживающих, наоборот, выше, чем у тех, кто ни за кем не ухаживает, но число рабочих часов ниже. Похожие результаты были получены и в других более поздних исследованиях (Johnson, Lo Sasso 2000; Heitmueller, Inglis 2004; Fevang, Kverndokk, Roed 2008; Carmichael, Charles, Hulme 2010).

Так же, как и в случае с безработными, сокращение рабочих часов в основном ощутимо среди тех, кто осуществляет уход в течение 20 и более часов в неделю; в некоторых странах (Австралия, Великобритания) эффект проявляется уже среди тех, кто осуществляет родственный уход в течение 10 и больше часов в неделю. Также стоит отметить, что если родственным уходом занимаются женщины, они чаще отказываются от занятости, в то время как мужчины чаще сокращают рабочие часы (Johnson, Lo Sasso 2000; Carmichael, Charles 2003; Heitmueller 2007).

За снижением возможностей зарабатывать следует и то, что в целом материальное положение домохозяйств, в которых есть ухаживающие и те, за кем ухаживают, хуже, так как происходит двойная потеря дохода (не работает тот, кто потерял способность к самообслуживанию и меньше работает, или не работает тот, кто ухаживает). Кроме того, могут возникать дополнительные траты на адаптацию жилища, специальную диету, различные приспособления и оборудование, специальную одежду и обувь и так далее (Rodrigues et al. 2013).

Таким образом, можно говорить, что предоставление долговременного ухода оказывает прямое влияние на факт занятости, в частности потому, что рынок труда не предоставляет достаточного количества рабочих мест с гибким рабочим графиком. 
Так, по данным Обследования рабочей силы, проведенного Росстатом в 2019 г., в России среди занятых в возрасте от 15 лет и старше лишь менее 3\% работали не более 20 часов в неделю. В то же время на принятие решений относительно ухода и поведения на рынке труда влияют социально-демографические характеристики людей, осуществляющих родственный уход. Чаще для роли ухаживающего выбирают членов семьи в более старших возрастах и с более низким уровнем образования, так как у них меньше возможностей на рынке труда и их занятость может приносить меньше трудового дохода (Colombo et al. 2011; Bauer, Sousa-Poza 2015). В результате всего вышесказанного можно сделать предположение, что родственный уход повышает риски бедности для лиц, ухаживающих за пожилыми и людьми с ограниченными возможностями здоровья.

В условиях увеличения доли работающих женщин (по данным сборника Росстата "Рабочая сила, занятость и безработица в России" (Росстат 2008; Росстат 2018) с 2000 по 2017 г. в России уровень занятости среди женщин увеличился с 53,8 до 60,1\%) уход за членами семьи в рамках домохозяйства становится более проблематичным, так как возникают сложности в сочетании трудовой занятости и обеспечения ухода. В связи с этим повышается важность внимания к данному вопросу со стороны государства, в том числе создания условий для получения профессионального ухода, системы субсидий и стимулирующих выплат для членов семей, занятых уходом, обеспечения на рынке труда политики, дающей возможность сочетать занятость и уход за родственниками, а также принятия других мер, призванных в совокупности решить данную проблему.

Еще одним негативным эффектом домашнего ухода является ухудшение душевного здоровья лиц, осуществляющих родственный уход, и их здоровья в целом, что также влияет на увеличение рисков бедности. Как правило, родственники и друзья, сталкиваясь с необходимостью осуществлять уход за своим близким человеком, не подготовлены к этому. Они не знают, как ухаживать; как разобраться в тех возможностях, которые предоставляет государственная система социального обслуживания, здравоохранения и другие ведомства; какой прогноз течения болезни или того состояния, в котором находится человек; как справиться с психологической нагрузкой, связанной с заботой о тяжело болеющем родственнике, что повышает тревожность и приводит к депрессии (Brodsky, Habib, Hirschfeld 2003). Исследование по Великобритании показало, что около половины людей, осуществляющих уход, столкнулись с ухудшением физического здоровья (болями в спине), также около половины лечились от заболеваний, вызванных стрессом, после того, как стали ухаживать за близким (Henwood 1998). Однако не все авторы подтверждают, что осуществление ухода ухудшает физическое здоровье (Schulz et al. 1995; Department of Health... 2000). Панельное исследование по США показало, что длительность ухода оказывает значимое влияние на физическое состояние человека, есть существенная разница в здоровье между теми, кто только начал ухаживать за близкими и кто делает это на протяжении длительного периода времени (Coe, Van Houtven 2009). Кроме того, интересный аспект подчеркнут в (Rodrigues et al. 2013): возможна обратная зависимость, люди с более слабым здоровьем более вероятно решат отказаться от работы и посвятить время уходу за близким человеком.

Ряд исследователей (Neundorfer 1991; Schulz et al. 1995; Schulz 2000; Toseland, Smith, McCallion 2001; Rodrigues et al. 2013) отмечают, что у ухаживающих часто возникают 
психологические проблемы (раздражительность, депрессия, прием психотропных препаратов и др.).

Анализ данных обследования здоровья, старения и выхода на пенсию в Европе (the Survey of Health, Ageing and Retirement in Europe (SHARE)), а также панельных данных по США показывает, что осуществляющие уход чаще страдают от психических расстройств (Coe, van Houtven 2009; Colombo et al. 2011).

Негативные эффекты на здоровье значимы для отдельных групп ухаживающих, в том числе пожилых супругов (Schulz et al. 1995), совместно проживающих (Rodrigues et al. 2013), а также тех, кто тратит на уход значительное время и усилия (Schulz, Sherwood 2008; Colombo et al. 2011). Однако важную роль может играть то, какие психические проблемы были у человека до того, как он начал ухаживать за близким, а также важно отличать эффект, который оказывает на здоровье именно осуществление ухода за близким от влияния самого факта того, что близкий человек находится в плохом состоянии здоровья и страдает (Schulz, Sherwood 2008). Кроме того, наличие родственника, за которым необходим уход, особенно проживающего совместно, приводит к риску изолированности от своего социального окружения (van Houtven et al. 2020, Gérain, Zech 2019).

Основываясь на проведенном обзоре зарубежных исследований, в рамках данной статьи мы решили проанализировать, как влияет осуществление ухода за пожилыми родственниками и родственниками с инвалидностью на социально-экономическое положение ухаживающих в России, в частности, рассмотреть влияние ухода на занятость, здоровье и материальное положение ухаживающих.

\section{МЕТОДЫ}

Источниками информации о лицах, осуществляюих родственный уход за пожилыми и людьми с ограниченными возможностями здоровья, в России служат данные обследований населения. К сожалению, Росстат не проводит детализированных обследований, полностью посвященных теме родственного ухода за пожилыми и инвалидами, однако в отдельных обследованиях населения, таких как «Комплексное наблюдение условий жизни населения» и «Выборочное наблюдение качества и доступности услуг в сферах образования, здравоохранения и социального обслуживания, содействия занятости населения» затрагиваются вопросы нуждаемости в уходе.

На основании данных «Комплексного наблюдения условий жизни населения» (далее - КОУЖ) Росстата существует возможность создать довольно подробный социальноэкономический портрет лиц, занимающихся уходом за родственниками. В частности, в КОУЖ задаются вопросы о том, входит ли в повседневные дела респондентов уход за другим лицом из-за нетрудоспособного возраста, болезни и нетрудоспособности и сколько часов в неделю они проводят, ухаживая за этим человеком. Помимо этого, в обследовании присутствуют вопросы о социально-демографических характеристиках респондента и характеристиках домохозяйства, типе населенного пункта и региона его проживания. 
Оценка социально-экономических последствий родственного ухода за пожилыми для ухаживающих, представленная в данной статье, была сделана на данных КОУЖ, проведенного Росстатом в 2018 г. во всех субъектах Российской Федерации и охватившего 60 тыс. домашних хозяйств. Результаты обследования репрезентативны как в целом по России, так и по городским и сельским поселениям с различной численностью населения и отдельным социально-демографическим группам населения.

В качестве переменной, идентифицирующей индивида, осуществляющего родственный уход, была взят ответ на вопрос: «Входит ли в круг ваших ежедневных занятий уход (без оплаты) за другим лицом (другими лицами), которое нуждается в особой помощи из-за престарелого возраста, болезни или нетрудоспособности? Это лицо (или эти лица) может проживать как в Вашем домохозяйстве, так и в другом месте». Кроме того, в ходе анализа учитывали интенсивность оказываемого ухода, которую определяли на основании ответа на вопрос: «Примерно, сколько часов в неделю Вы проводите, ухаживая за этим человеком (этими людьми)?». Анализ был направлен на выявление различий в распространенности ухода среди различных групп населения, в уровне и интенсивности занятости на рынке труда лиц, осуществляющих уход, в том числе с учётом интенсивности ухода, состояния их здоровья и уровня доходов.

При анализе взаимосвязи между фактом, а также интенсивностью ухода за пожилыми родственниками и различными социально-демографическими характеристиками ухаживающих лиц (пол, возраст, место проживания, наличие детей, статус и характеристики их занятости, состояние их здоровья и материальное положение) проводили проверку с помощью критерия Хи-квадрат. Представленные в работе результаты являются значимыми по данному критерию на 1\%-ном уровне значимости. Кроме того, для проверки значимости различий между группами дополнительно проводили тест Стьюдента на равенство средних для двух независимых выборок. Описанные в статье различия между группами являются значимыми на 5\%-ном уровне значимости.

\section{СОЦИАЛЬНО-ДЕМОГРАФИЧЕСКИЙ ПОРТРЕТ УХАЖИВАЮЩИХ ЛИЦ И ИНТЕНСИВНОСТЬ ИХ УХОДА}

Исследование, выполненное на данных КОУЖ 2018 г. показало, что среди лиц старше 18 лет около $6 \%$ осуществляют уход за пожилыми родственниками и инвалидами. Существенных различий по доле занятых уходом среди городских и сельских населенных пунктов не наблюдается. Также между федеральными округами в основном есть лишь небольшие различия в доле лиц, осуществляющих уход за пожилыми и инвалидами. Наибольшее различие между Северо-Кавказским федеральным округом, где доля осуществляющих уход за пожилыми родственниками и инвалидами самая высокая, и Уральским федеральным округом, где она самая низкая (9 и 5\% соответственно). Относительно высокая доля лиц, самостоятельно осуществляющих уход за своими пожилыми родственниками в Северо-Кавказском федеральном округе, может объясняться традиционным укладом жизни народов Кавказа. Среди осуществляющих уход за пожилыми и инвалидами доля женщин выше, чем среди тех, кто такой уход не осуществляет: $67 \%$ против 55\%. При этом более $65 \%$ осуществляющих уход за пожилыми 
и инвалидами - женщины. То, что уход за пожилыми родственниками чаще ложится на плечи женщин, согласуется и с выводами зарубежных исследователей (Rodrigues et al. 2013).

Доля лиц, осуществляющих уход, повышается с возрастом и является максимальной для возрастной группы 50-59 лет (10\%, среди женщин - 12\%, среди мужчин - 7\%), а затем постепенно снижается. Поскольку уход осуществляется, как правило, за своими родителями, родителями супруга или бабушками и дедушками в тот момент, когда их состояние здоровья существенно ухудшается (а это происходит обычно после наступления пенсионного возраста), то и осуществляющие уход уже достигают предпенсионного возраста. Уход за пожилыми и инвалидами чаще совершают люди с выросшими детьми старше 18 лет, однако и среди лиц с детьми до 18 лет доля тех, кто ухаживает за пожилыми родственниками и родственниками с инвалидностью, достаточно существенна и составляет $4-5 \%$.

Значимых различий в уровне образования между респондентами, осуществляющими и не осуществляющими уход, выявлено не было, что свидетельствует о том, что люди как с высоким, так и с низким уровнем образования могут в равной степени столкнуться с необходимостью осуществлять уход за своими пожилыми родственниками.

Наименее интенсивный уход (менее 1 часа в день) несколько чаще распространен в городах, чем в сельских населенных пунктах (18\% против 12\%). В городах-миллионниках наблюдается наиболее низкая доля тех, кто осуществляет наиболее интенсивный уход: 4-6 часов $(10 \%)$ и более 6 часов $(6 \%)$. В то же время в населенных пунктах с числом жителей менее 50 тыс. доля осуществляющих наиболее интенсивный уход выше и составляет соответственно 16 и 11\%. Если в крупных городах рынок негосударственных услуг по уходу является более развитым и их жители имеют более высокий доход и могут себе позволить оплатить услуги по уходу за своими пожилыми родственниками, то в небольших городах и сельских населенных пунктах рынок услуг по уходу зачастую отсутствует и, кроме того, невысокие доходы жителей не позволяют им оплачивать такие услуги.

Выше всего доля тех, кто осуществляет самый интенсивный уход (от 6 часов и более в день) в Северо-Западном и Приволжском федеральных округах (более $11 \%$ ), а ниже всего - в Центральном федеральном округе $(5,6 \%)$. Самая низкая доля осуществляющих уход менее 1 часа в день в Уральском, Южном и Северо-Кавказском федеральных округах (менее 14\%), а самая высокая - в Дальневосточном (20\%).

Во всех группах интенсивности ухода доля женщин выше, чем доля мужчин. Однако, если в группе наименее интенсивного ухода (менее 1 часа в день) доля женщин составляет $57 \%$, то, начиная со времени ухода от 2 часов в день, эта доля возрастает до $71 \%$. Самая существенная разница наблюдается в группе с наибольшей интенсивностью ухода (от 6 часов и более в день), где доля женщин составляет 78\%, а доля мужчин - всего 22\%.

Среди осуществляющих уход более $20 \%$ имеют детей до 18 лет, причем $8 \%$ - двух и более детей до 18 лет. Лица, имеющие детей, несут двойную нагрузку, так как ухаживают не только за детьми, но и за родственниками, нуждающимися в уходе. 
В самых молодых группах (до 30 лет) наиболее высока доля ухаживающих менее 2 часов в день (более 60\%). В возрасте 18-24 года уход от 6 часов и более в день осуществляют менее 3\% ухаживающих. В 40-44 года и 45-49 лет заметно падает доля ухаживающих менее 1 часа в день и растет доля тех, кто ухаживает более продолжительное время, в том числе наблюдается достаточно сильный рост доли ухаживающих 4,0-5,9 часов (11\%). С увеличением возраста растет доля лиц, несущих самую высокую нагрузку (более 6 часов в день), что является свидетельством того, что уход в основном осушествляется за супругами в пожилом возрасте, состояние здоровья которых ухудшается. Достаточно резкий рост происходит в группе с 55 лет, что говорит о том, что возможность интенсивнее ухаживать за родственниками появляется у тех, кто выходит на пенсию, что совпадает также и с ростом нуждаемости в уходе у супругов и пожилых родителей (11\% ухаживают от 6 часов и более; 16\% - 4,0-5,9 часов). Следующий существенный рост доли ухаживающих от 6 часов и более заметен в группе 65-69 летних (18\%), что может объясняться ухудшением состояния здоровья подопечных в связи с их преклонным возрастом. Самая высокая степень интенсивности ухода приходится на группы самого старшего возраста от 75 лет (26\% ухаживают от 6 часов и более в день).

Среди лиц трудоспособного возраста, ухаживающих за пожилыми родственниками 6 часов и более в день, доля людей с высшим образованием существенно ниже, чем среди тех, кто ухаживает менее 1 часа в день (29\% против 41\%). Такая же тенденция присутствует и среди отдельных десятилетних возрастных групп. Так, например, среди респондентов 3039 лет, осуществляющих наиболее интенсивный уход, доля лиц с высшим образованием $35 \%$, в то время как среди осуществляющих наименее интенсивный уход $-55 \%$. Это может свидетельствовать о том, что не имеющие высшего образования чаще осуществляют затратный по времени уход, поскольку у них нет возможности оплатить услуги по уходу.

\section{ЗАНЯТОСТЬ ЛИЦ, ОСУЩЕСТВЛЯЮЩИХ УХОД}

Лица в трудоспособном возрасте, осуществляющие уход, несколько реже имеют работу, чем те, кто такой уход не осуществляет (76\% против 80\%). Так, например, среди ухаживающих за родственниками респондентов в возрасте от 30 до 39 лет доля работающих на 8 п.п. ниже, чем среди тех, кто не ухаживает (77\% против 85\%), а в возрасте от 40 до 49 лет доля работающих ниже на 7 п.п. (82\% против 89\%). В то же время среди лиц старше трудоспособного возраста до 70 лет ситуация противоположная: ухаживающие чаще имеют работу, чем те, кто не ухаживает (35\% против $28 \%$ ), что может быть связано с тем, что уход, как правило, осуществляется в раннем пенсионном возрасте, когда пенсионеры еще не прекратили работать. Респонденты трудоспособного возраста, имеющие работу и осуществляющие уход за пожилыми и инвалидами, несколько чаще работают менее 40 часов в неделю (19\% против 14\%). В частности, в возрасте от 30 до 39 лет среди ухаживающих доля тех, кто работает менее 40 часов в неделю, составляет 19\%, в то время как среди лиц этого возраста, не осуществляющих уход, - 14\%, среди 40-49-летних - 17$14 \%$ соответственно.

Реже имеют работу респонденты в трудоспособном возрасте, осуществляющие уход и не имеющие высшего образования: среди них работу имеют $70 \%$ респондентов против 
$76 \%$ среди лиц трудоспособного возраста без высшего образования, не осуществляющих уход. В то же время среди респондентов трудоспособного возраста с высшим образованием как осуществляющих, так и не осуществляющих уход, уровень занятости примерно одинаковый и составляет 86-87\%. Кроме того, лица в трудоспособном возрасте, ухаживающие за своими пожилыми родственниками и не имеющие высшего образования, чаще работают менее 40 часов в неделю (44\% против $35 \%$ среди лиц трудоспособного возраста без высшего образования, не осуществляющих уход).

Таким образом, проведенный анализ показал, что люди трудоспособного возраста, ухаживающие за пожилыми родственниками, реже имеют работу и чаще работают неполный рабочий день. В первую очередь это касается не имеющих высшего образования.

В ходе анализа не удалось найти подтверждения тому, что необходимость ухода за пожилыми родственниками и инвалидами приводит к переходу от занятости на предприятии к самозанятости или частному предпринимательству (среди осуществляющих и не осуществляющих уход доля тех, кто работает самозанятыми или индивидуальными предпринимателями, существенно не различается и составляет 6\%), а также к переходу к неформальной занятости на основе устных договоренностей (среди осуществляющих и не осуществляющих уход доля работающих на условиях устных договоренностей без оформления трудового договора существенно не различается и составляет $6 \%$ ). Кроме того, не было найдено подтверждений тому, что сам по себе факт ухода индивида за престарелыми родственниками и инвалидами (без учета интенсивности ухода) негативно влияет на его среднедушевые доходы (распределение респондентов, осуществляющих и не осуществляющих уход, по доходам в зависимости от величины прожиточного минимума существенно не различается).

Доля лиц, занятых на рынке труда, значительно сокращается с ростом интенсивности ухода: 75\% работающих среди тех, кто ухаживает менее 1 часа в день, против $26 \%$ среди тех, кто ухаживает от 6 часов и более в день. Эта тенденция сохраняется, если рассматривать отдельные возрастные группы: среди 30-39-летних эти значения составляют соответственно 79 и 49\%; среди 60-69-летних - 40 и $8 \%$.

Интенсивность ухода существенным образом влияет на возможность работать для людей в трудоспособном возрасте $(83 \%$ работающих в группах с наименьшей интенсивностью ухода против $43 \%$ - с наибольшей). Также с ростом интенсивности ухода снижается доля работающих в возрасте старше трудоспособного (40\% против 13\%). Доля работающих выше среди респондентов трудоспособного возраста, осуществляющих наименее интенсивный уход и имеющих высшее образование (88\% против $65 \%$ среди тех, кто осуществляет наиболее интенсивный уход). Аналогичная ситуация наблюдается и среди лиц трудоспособного возраста без высшего образования: доля работающих также снижается при росте интенсивности ухода (80\% против 34\%). Таким образом, независимо от уровня образования респондента при повышении интенсивности его ухода за пожилым родственником возможности для его занятости ухудшаются.

Рост нагрузки по уходу влияет на уменьшение числа часов работы у лиц, осуществляющих уход (доля работающих от 40 часов в неделю и более падает с 80 до $62 \%$ в группе самого интенсивного ухода). Кроме того, с ростом интенсивности нагрузки растет 
доля работающих на предприятиях со статусом юридического лица, в первую очередь, за счет снижения доли занятых на предприятиях индивидуальных предпринимателей с 13 до 7\%. Также с ростом интенсивности ухода происходит снижение доли работающих полный рабочий день - с 88 до 79\% и рост доли работающих неполный рабочий день - -c 3 до 10\%.

Таким образом, более интенсивный и затратный по времени уход за пожилыми родственниками сокращает возможности занятости, в том числе занятости полный рабочий день.

\section{СОСТОЯНИЕ ЗДОРОВЬЯ ЛИЦ, ОСУЩЕСТВЛЯЮЩИХ УХОД}

Ухаживающие за родственниками лица в возрасте от 18 до 60 лет хуже оценивают состояние своего здоровья, по сравнению с не осуществляющими уход: доля оценивающих свое здоровье как «хорошее» или «очень хорошее» на 4-10 п.п. ниже. Эта тенденция сохраняется внутри отдельных возрастных групп: среди лиц 18-29 лет, осуществляющих уход, доля тех, кто оценивает свое здоровье как «хорошее» и «очень хорошее», составляет $73 \%$, в то время как среди лиц этого же возраста, но не осуществляющих уход, - уже 80\%; среди 30-39-летних - 60 и 68\% соответственно; среди 40-49-летних - 41 и 51\%; среди 5059-летних - 21 и 24\%. В то же время среди лиц в возрасте старше 60 лет самооценка здоровья ухаживающих и не ухаживающих за пожилыми родственниками и родственниками с инвалидностью существенным образом не различается.

Кроме того, ухаживающие за своими родственниками люди в возрасте от 30 до 60 лет чаще (на 5-6 п.п.) имеют хронические заболевания по сравнению с не осуществляющими уход: например, среди лиц 30-39 лет, осуществляющих уход, доля тех, кто имеет хронические заболевания, составляет $15 \%$, в то время как среди лиц этого же возраста, но не осуществляющих уход, - лишь 10\%; среди 40-49-летних - 21 и 16\% соответственно; среди 50-59-летних - 37 и 31\%. В то же время среди ухаживающих и не ухаживающих в возрасте старше 60 лет доля тех, кто имеет хронические заболевания, существенно не различается.

Интенсивность ухода сильно влияет на здоровье ухаживающих. В группе самого интенсивного ухода своё здоровье оценивают как «хорошее или очень хорошее» лишь $41 \%$ респондентов трудоспособного возраста и 4\% старше трудоспособного возраста против 48 и $13 \%$ в соответствующих группах с уходом менее 1 часа в день. Негативное влияние интенсивности ухода на здоровье осуществляющих уход наблюдается и в разрезе десятилетних возрастных групп. Так, например, среди лиц 30-39 лет, осуществляющих наиболее интенсивный уход (от 6 часов и более), доля оценивающих свое здоровье как «хорошее или очень хорошее» составляет 3\%, в то время как среди ухаживающих менее 1 часа в день $-43 \%$; в группе $50-59$ лет -1 и $12 \%$ соответственно.

Кроме того, в группе осуществляющих самый интенсивный уход (от 6 часов и более) наиболее высока доля имеющих хронические заболевания (42\%). Эта тенденция также подтверждается в разрезе десятилетних возрастных групп. 
Таким образом, ухаживающие хуже оценивают состояние своего здоровья и чаще имеют хронические заболевания. Это может быть связано, в том числе, с тем, что лица, осуществляющие уход за пожилыми родственниками, имеют меньше свободного времени для того, чтобы заниматься своим здоровьем, проходить профилактические медицинские осмотры. Кроме того, интенсивный уход, который может предполагать действия, связанные с подъемом и перемещением маломобильного человека, может негативно сказываться на физическом здоровье ухаживающих, например, повышать риски развития у них заболеваний суставов, позвоночника и сосудов.

\section{МАТЕРИАЛЬНОЕ ПОЛОЖЕНИЕ ЛИЦ, ОСУЩЕСТВЛЯЮЩИХ УХОД}

Распределение осуществляющих и не осуществляющих уход за пожилыми родственниками и инвалидами по уровню среднедушевого дохода семьи в зависимости от величины прожиточного минимума существенным образом не различается как в группе трудоспособных, так и в группе старше трудоспособного возраста. Таким образом, не было найдено подтверждения тому, что факт ухода за престарелыми родственниками и инвалидами негативно влияет на среднедушевые доходы домохозяйства.

Около половины всех осуществляющих уход имеют среднедушевой доход от 1 до 2 величин прожиточного минимума. При этом самая высокая доля имеющих доходы ниже величины прожиточного минимума среди тех, кто ухаживает от 6 часов и более в день (24\%). Также в группе самого интенсивного ухода самая низкая доля тех, кто имеет доходы в размере двукратной величины прожиточного минимума и выше (10\%). Для сравнения: в группе ухаживающих менее 1 часа в день доля людей с доходом в размере двукратной величины прожиточного минимума и выше составляет уже $30 \%$.

В распределении лиц трудоспособного возраста, осуществляющих уход различной степени интенсивности, наблюдается резкий рост доли имеющих среднедушевой доход ниже величины прожиточного минимума с ростом интенсивности ухода. В группе с самой интенсивной нагрузкой (от 6 часов в день и более) уже $42 \%$ малоимущих (против $21 \%$ в группе с наименее интенсивным уходом). Среди респондентов старше трудоспособного возраста при увеличении интенсивности ухода можно отметить резкое сокращение доли имеющих среднедушевой доход свыше двукратной величины прожиточного минимума (с 22 до 6\%). Тенденция увеличения доли малоимущих среди лиц трудоспособного возраста, осуществляющих уход за пожилыми родственниками, при повышении интенсивности ухода наблюдается и в разрезе отдельных групп, сформированных по уровню образования. Так, например, среди лиц трудоспособного возраста с высшим образованием, ухаживающих за пожилыми родственниками менее 1 часа в день, доля малоимущих составляет $11 \%$, в то время как среди осуществляющих наиболее интенсивный уход - 33\%; со средним профессиональным образованием (по программе подготовке рабочих) - 29 и $49 \%$ соответственно.

Таким образом, можно сделать вывод, что интенсивный уход за пожилыми родственниками ухудшает материальное положение ухаживающих и повышает риски их бедности. 


\section{СРАВНЕНИЕ ХАРАКТЕРИСТИК ЗАНЯТОСТИ ЛИЦ, ОСУЩЕСТВЛЯЮЩИХ УХОД, В РОССИИ И В ЗАРУБЕЖНЫХ СТРАНАХ}

Данные ОЭСР свидетельствуют, что в среднем по странам ОЭСР 13,5\% лиц старше 50 лет осуществляли уход за своими пожилыми родственниками. В России доля ухаживающих по данным КОУЖ Росстата меньше - 7\% (рисунок 1).

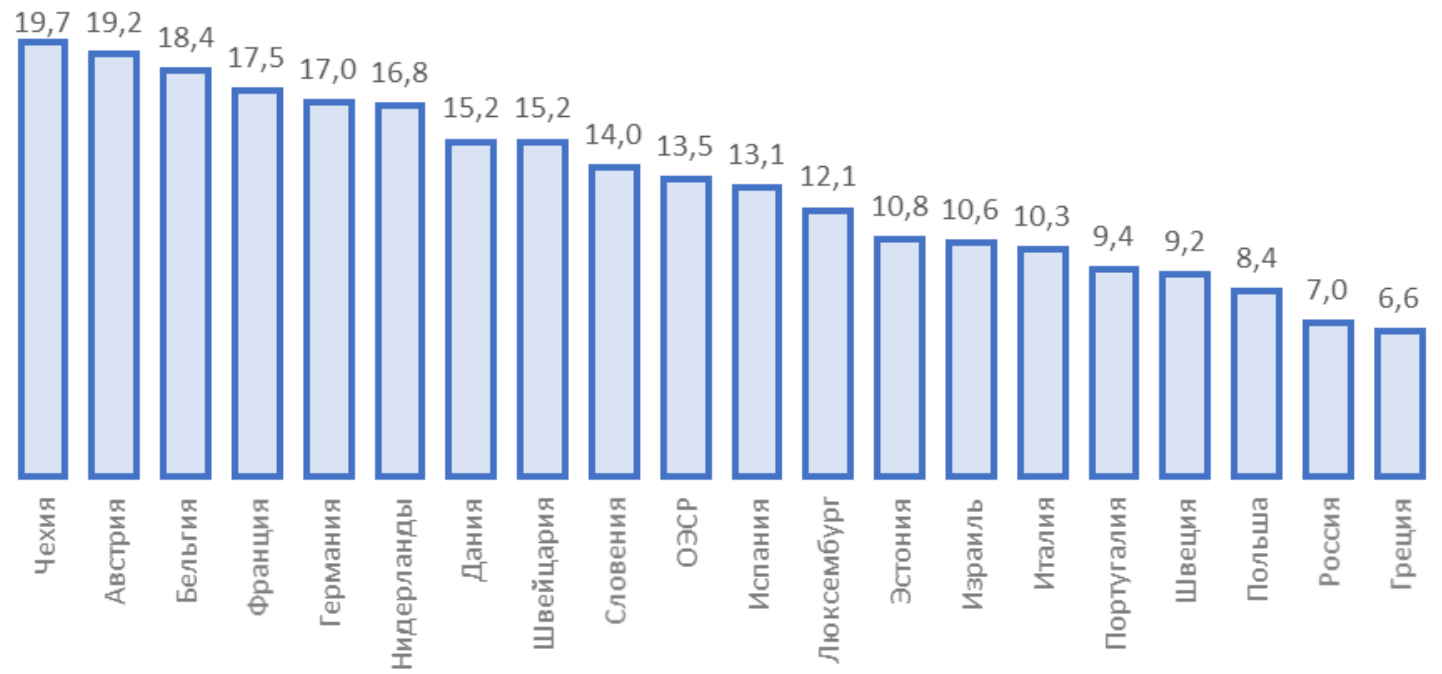

Рисунок 1. Доля лищ, осуществляющих родственный уход, \%

Источник: Данные ОЭСР за 2005-2010 г2. (Colombo et al. 2011); расчеты авторов на данных КОУЖ2018 Poccmama.

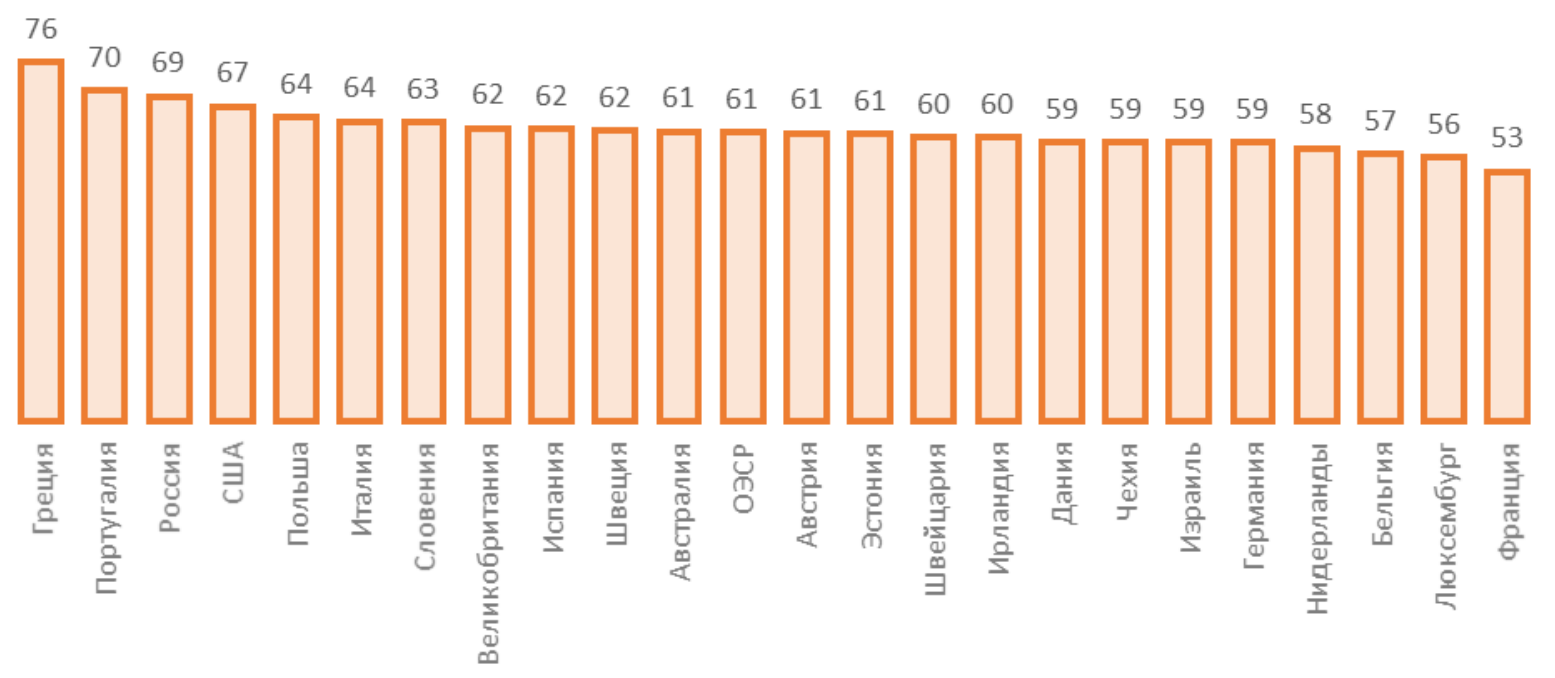

Рисунок 2. Доля женщин среди лиц 50+, осуществляющих уход, в России и ОЭСР, \%

Источник: Данные ОЭСР за 2005-2010 г2. (Colombo et al. 2011); расчеты авторов на данных КОУЖ2018 Poccmama.

При этом надо отметить слабую сопоставимость опросов по теме ухода, проводимых в различных странах, с методологической точки зрения: респонденты могут понимать под уходом различные виды деятельности и могут не рассматривать в качестве ухода поддержку, осуществляемую изредка, например, еженедельную покупку и доставку на дом 
продуктов и товаров. Это подтверждается тем, что в России около половины респондентов, отметивших, что ухаживают за родственниками, сказали, что они ухаживают более 20 часов в неделю, в то время как в среднем по странам ОЭСР доля таких лиц лишь 33\%. Таким образом, интенсивность ухода в России выше.

В среднем по странам ОЭСР 61\% ухаживающих за пожилыми родственниками женщины, в то время как в России эта доля еще выше - 69\% (рисунок 2).

В среднем по странам ОЭСР, как и в России, среди ухаживающих за пожилыми родственниками уровень занятости несколько ниже, чем среди тех, кто такой уход не осуществляет (рисунок 3). Наиболее значимые различия наблюдаются в Польше, где доля работающих среди осуществляющих уход составляет лишь $34 \%$, в то время как среди не занятых уходом $-60 \%$.

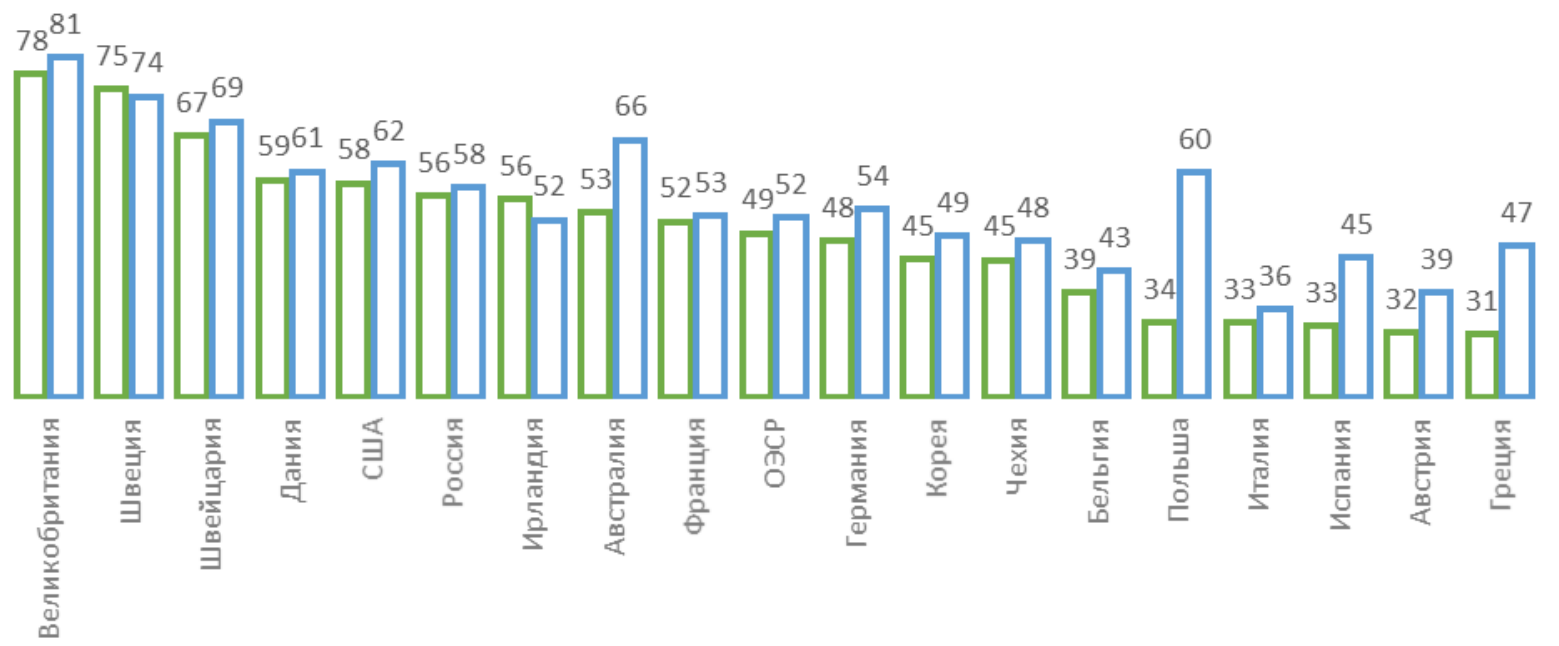

口Доля работающих среди ухаживающих, \% Доля работающих среди неухаживающих, \%

Рисунок 3. Доля работающих среди лиц 50-64 лет, осуществляющих и не осуществляющих уход, \%

Источник: Данные ОЭСР за 2005-2010 г2. (Colombo et al. 2011); расчеты авторов на данных КОУЖ2018 Poccmama.

\section{Выводы}

Потребность в уходе усиливается с возрастом при ухудшении состояния здоровья. В связи с этим необходимость ухода за своими ближайшими родственниками (например, супругом или родителями) возникает, как правило, в предпенсионном и пенсионном возрасте. В то же время пятая часть осуществляющих родственный уход имеет несовершеннолетних детей и испытывает двойную нагрузку по уходу как за своими детьми, так и за пожилыми родственниками.

Если в крупных городах рынок негосударственных услуг по уходу является более развитым и их жители имеют более высокий доход и могут себе позволить оплатить услуги по уходу, то в небольших городах и селах жители чаще осуществляют уход за пожилыми 
родственниками самостоятельно. Как и в других странах, в России за пожилыми родственниками чаще ухаживают именно женщины, особенно если требуется значительный по времени уход.

Лица трудоспособного возраста, ухаживающие за пожилыми родственниками, реже имеют работу и чаще работают неполный рабочий день. В первую очередь это касается не имеющих высшего образования и не имеющих возможности оплатить услуги по уходу. Более интенсивный и затратный по времени уход за пожилыми родственниками сокращает возможности занятости осуществляющих уход, в том числе их занятости полный рабочий день.

Ухаживающие хуже оценивают состояние своего здоровья и чаще имеют хронические заболевания, что может быть связано с отсутствием у них времени для того, чтобы заниматься своим здоровьем, а также с существенными физическими нагрузками, возникающими при уходе за маломобильными людьми в старческом возрасте или с инвалидностью.

Анализ показал, что интенсивный уход за пожилыми родственниками и инвалидами, снижая возможности занятости, ухудшает материальное положение ухаживающих. Среди людей трудоспособного возраста, осуществляющих интенсивный родственный уход, выше доля бедных и крайне бедных и ниже доля лиц со среднедушевым доходом выше двукратной величины прожиточного минимума.

Это свидетельствует о необходимости создания в России комплексной системы мер, направленных на поддержку родственников, осуществляющих уход, включая меры по сохранению занятости и развитию гибких форм занятости на рынке труда, расширению охвата лиц, нуждающихся в уходе, программами временного и дневного ухода, оказанию консультационной поддержки родственников, осуществляющих уход, и организации их обучения навыкам ухода как в очной, так и в дистанционной форме. Указанные меры поддержки родственников, осуществляющих уход, применяются в зарубежных странах и позволяют улучшить положение ухаживающих, а пожилым людям и инвалидам, нуждающимся в уходе, дают возможность как можно дольше оставаться дома в привычных условиях и получать при этом качественный уход.

Проведенный в рамках данной работы анализ и полученные результаты позволили сформировать выводы о взаимосвязях родственного ухода и ряда характеристик ухаживающих лиц. В ходе дальнейших исследований следует провести углубленный анализ выявленных взаимосвязей, установить причинно-следственные связи между фактом ухода и характеристиками ухаживающих. В частности, с использованием регрессионного анализа на основе панельных данных можно изучить, могут ли какие-либо характеристики индивида повышать, при прочих равных условиях, вероятность его ухода в будущем за пожилым родственником, а также оказывает ли факт ухода за пожилым родственником негативное влияние на будущее материальное положение ухаживающего, статус его занятости и состояние его здоровья. 


\section{ЛИТЕРАТУРА}

Росстат (2018). Рабочая сила, занятость и безработица в России - 2018 г. URL: https://gks.ru/bgd/regl/B18_61/Main.htm (данные загружены 29.06.2020).

Росстат (2008). Экономическая активность населения России - 2008 г. URL: https://gks.ru/bgd/regl/B08_61/Main.htm (данные загружены 29.06.2020).

Attias-Donfut C., Ogg J., Wolff F. (2005). European patterns of intergenerational financial and time transfers. European Journal of Ageing, 2, 161-173. DOI: https://doi.org/10.1007/s10433-005-0008-7

Bauer M.J., Sousa-Poza A. (2015). Impacts of Informal Caregiving on Caregiver Employment, Health, and Family. Population Ageing, 8 (3), 113-145. DOI: https://doi.org/10.1007/s 12062015-9116-0

Brodsky J., Habib J., Hirschfeld M. (Eds.). (2003). Key policy issues in long-term care. Geneva: WHO. URL: https://www.who.int/chp/knowledge/publications/policy_issues_ltc.pdf

Brown M.R., Brown L.S. (2014). Informal caregiving: A reappraisal of effects on caregivers. Social Issues and Policy Review, 8, 74-102. https://doi.org/10.1111/sipr.12002

Carmichael F., Charles S. (1998). The labour market costs of community care. Journal of Health Economics, 17, 747-765. DOI: https://doi.org/10.1016/s0167-6296(97)00036-2

Carmichael F., Charles S. (2003). The Opportunity Costs of Informal Care: Does Gender Matter? Journal of Health Economics, 22 (5), 781-803. DOI: https://doi.org/10.1016/S01676296(03)00044-4

Carmichael F., Charles S., Hulme C. (2010). Who will care? Employment participation and willingness to supply informal care. Journal of Health Economics, 29, 182-190. DOI: https://doi.org/10.1016/j.jhealeco.2009.11.003

Coe N.B., van Houtven C.H. (2009). Caring for mom and neglecting yourself? The health effects of caring for an elderly parent. Journal of Health Economics, 18(9), 991-1010. DOI: https://doi.org/10.1002/hec.1512

Colombo F., Llena-Nozal A., Mercier J., Tjadens F. (2011). Help Wanted? Providing and Paying for Long-Term Care. OECD Health Policy Studies. Paris: OECD Publishing. DOI: https://doi.org/10.1787/9789264097759-en

Denham A.M.J., Wynne O., Baker A.L., Spratt N.J., Turner A., Magin P. et al. (2019) "This is our life now. Our new normal": A qualitative study of the unmet needs of carers of stroke survivors. PLoS ONE, 14(5). DOI: https://doi.org/10.1371/journal.pone.0216682

Department of Health and Social Care, UK (2000). A National Strategy for Carers. URL: https://www.gov.uk/government/news/a-national-strategy-for-carers

Fevang E., Kverndokk S., Roed K. (2008). Informal care and labor supply. IZA Discussion Paper Series No. 3717. Bonn: IZA. URL: http://ftp.iza.org/dp3717.pdf

Gérain P., Zech E. (2019). Informal Caregiver Burnout? Development of a Theoretical Framework to Understand the Impact of Caregiving. Frontiers in Psychology, 10, 1748. DOI: https://doi.org/10.3389/fpsyg.2019.01748

Greenwood N., Pound C., Brearley S., Smith R. (2019) A qualitative study of older informal carers' experiences and perceptions of their caring role. Maturitas, 124, 1-7. DOI: https://doi.org/10.1016/j.maturitas.2019.03.006 
Grundy E., Henretta J.C. (2006). Between elderly parents and adult children: a new look at the intergenerational care provided by the 'sandwich generation. Ageing and Society, 26, 707722. DOI: https://doi.org/10.1017/S0144686X06004934

Heitmueller A., Inglis K. (2004). Carefree? Participation and pay differentials for informal carers in Britain. IZA Discussion Paper Series No. 1273. Bonn: IZA. URL: http://ftp.iza.org/dp1273.pdf

Heitmueller, A. (2007). "The Chicken or the Egg? Endogeneity in Labour Market Participation of Informal Carers in England". Journal of Health Economics, 26(3).

DOI: https://doi.org/10.1016/j.jhealeco.2006.10.005

Henwood M. (1998). Ignored and Invisible? Carers Experience of the NHS. London: Carers' National Association.

Johnson R.W., Lo Sasso A.T. (2000). The Trade-Off between Hours of Paid Employment and Time Assistance to Elderly Parents at Mid-Life. Washington, DC: The Urban Institute. URL: https://www.urban.org/research/publication/trade-between-hours-paid-employment-andtime-assistance-elderly-parents-midlife/view/full_report

Katz S., Ford A.B., Moskowitz R.W., Jackson B.A., Jaffe M.W. (1963). Studies of Illness in the Aged: The Index of ADL: A Standardized Measure of Biological and Psychosocial Function. JAMA, 185 (12), 914-919. DOI: https://doi.org/10.1001/jama.1963.03060120024016

Lane P., McKenna H., Ryan A. (2003). Fleming P. The Experience of the Family Caregivers' Role: A Qualitative Study. Research and Theory for Nursing Practice, 17(2), 137-151(15). DOI: https://doi.org/10.1891/rtnp.17.2.137.53173

Latif E. (2013). Labour supply effects of informal caregiving in Canada. Canadian Public Policy, 32, 413-429. DOI: https://doi.org/10.3138/Q533-8847-3785-1360

Li S., Song L., Feldman M.W. (2009). Intergenerational support and subjective health of older people in rural China: a gender-based longitudinal study. Australasian Journal of Ageing, 28(2), 81-86. DOI: https://doi.org/10.1111/j.1741-6612.2009.00364.x

McGarrigle Ch., Kenny R.A. (2013). Profile of the sandwich generation and intergenerational transfers in Ireland. The Irish Longitudinal Study on Ageing. Dublin: Trinity College Dublin.

Neundorfer M.M. (1991). Family Caregiving of the Frail Elderly: Impact of Caregiving on their Health and Implications for Interventions. Community Health, 14, 48-58. DOI: https://doi.org/10.1097/00003727-199107000-00007

Niimi Y. (2016). The "Costs" of Informal Care: An Analysis of the Impact of Elderly Care on Caregivers' Subjective Well-being in Japan. Review of Economics of the Household, 14, 779-810. DOI: https://doi.org/10.1007/s11150-016-9333-1

Rodrigues R., Schulmann K., Schmidt A., Kalavrezou N., Matsaganis M. (2013). The indirect costs of long-term care. Research note 8. European Commission. URL: https://ec.europa.eu/social/BlobServlet?docId=11780\&langId=en

Schulz K. (2000). Handbook on Dementia Caregiving, Evidence-Based. Interventions for Family Caregivers. New York: Springer.

Schulz R. et al. (1995). Psychiatric and Physical Morbidity Effects of Dementia Caregiving:

Prevalence, Correlates, and Causes. Gerontolgist, 35, 771-791.

DOI: https://doi.org/10.1093/geront/35.6.771 
Schulz R., Sherwood P. (2008). Physical and mental health effects of family caregiving. Journal of American Nursing, 108, 23-27.

DOI: https://doi.org/10.1097/01.NAJ.0000336406.45248.4c

Toseland R.W., Smith G., McCallion P. (2001). Family Caregivers of the Frail Elderly. Prepared for the US Administration on Aging. Washington, DC: US Department of Health and Human Services.

Van den Berg B., Fiebig D.G., Hall J. (2014). Well-being losses due to care-giving. Journal of Health Economics, 35, 123-131. DOI: https://doi.org/10.1016/j.jhealeco.2014.01.008

Van Houtven C.,Carmichael F., Jacobs J, Coyte P.C. (2020). The Economics of Informal Care. Oxford University Press USA.

Viitanen T.K. (2010). Informal eldercare across Europe: estimates from the European Community Household Panel. Economic Analysis and Policy, 40, 149-178.

DOI: https://doi.org/10.1016/S0313-5926(10)50023-7 


\title{
THE EFFECT OF CARING FOR OLDER AND DISABLED RELATIVES ON THE EMPLOYMENT, HEALTH AND ECONOMIC STATUS OF CAREGIVERS
}

\author{
ELENA GRISHINA, ELENA TSATSURA
}

\begin{abstract}
In Russia, as in most other countries, it is the relatives who bear the main burden of caring for the elderly and disabled. Based on the survey data "Comprehensive Observation of the Living Conditions of the Population", the article analyses the effect of related care on the situation of caregivers. In particular, it has been shown that people of working age caring for relatives are less likely to have a job, more often work part-time or part-time, and are more likely to have a chronic illness and lower health self-assessment. Intensive care (more than 20 hours per week) increases the negative impact on employment and health selfassessment. Among people of working age who are providing intensive family care, the proportion of the poor and extremely poor is higher and the proportion of people with an average income of more than two subsistence levels is lower. This implies that a comprehensive system of measures aimed at supporting relatives providing care is needed - in particular, at preserving jobs, developing flexible forms of employment, expanding programs for temporary and day care, providing consulting support and organizing full-time and distance learning of caring skills.
\end{abstract}

Key words: career, long-term care, informal care, elderly social care.

\footnotetext{
Elena Grishina (grishina@ranepa.ru), Institute fOr Social ANALYSIS AND PREDiction RANEPA, RusSia.

Elena Tsatsura (tsatsura-ea@ranepa.ru), Institute for SocIAl ANALYSIS AND PREDiction RANEPA, RusSia.

THE ARTICLE WAS PREPARED AS PART OF THE RESEARCH WORK OF THE STATE ASSIGNMENT OF THE RANEPA.

DATE RECEIVED: APRIL 2020.
}

\section{REFERENCES}

Attias-Donfut C., Ogg J., Wolff F. (2005). European patterns of intergenerational financial and time transfers. European Journal of Ageing, 2, 161-173.

DOI: https://doi.org/10.1007/s10433-005-0008-7

Bauer M.J., Sousa-Poza A. (2015). Impacts of Informal Caregiving on Caregiver Employment, Health, and Family. Population Ageing, 8 (3), 113-145. DOI: https://doi.org/10.1007/s12062015-9116-0

Brodsky J., Habib J., Hirschfeld M. (Eds.). (2003). Key policy issues in long-term care. Geneva: WHO. URL: https://www.who.int/chp/knowledge/publications/policy_issues_ltc.pdf

Brown M.R., Brown L.S. (2014). Informal caregiving: A reappraisal of effects on caregivers. Social Issues and Policy Review, 8, 74-102. https://doi.org/10.1111/sipr.12002

Carmichael F., Charles S. (1998). The labour market costs of community care. Journal of Health Economics, 17, 747-765. DOI: https://doi.org/10.1016/s0167-6296(97)00036-2

Carmichael F., Charles S. (2003). The Opportunity Costs of Informal Care: Does Gender Matter? Journal of Health Economics, 22 (5), 781-803. DOI: https://doi.org/10.1016/S01676296(03)00044-4 
Carmichael F., Charles S., Hulme C. (2010). Who will care? Employment participation and willingness to supply informal care. Journal of Health Economics, 29, 182-190.

DOI: https://doi.org/10.1016/j.jhealeco.2009.11.003

Coe N.B., van Houtven C.H. (2009). Caring for mom and neglecting yourself? The health effects of caring for an elderly parent. Journal of Health Economics, 18(9), 991-1010. DOI: https://doi.org/10.1002/hec.1512

Colombo F., Llena-Nozal A., Mercier J., Tjadens F. (2011). Help Wanted? Providing and Paying for Long-Term Care. OECD Health Policy Studies. Paris: OECD Publishing. DOI: https://doi.org/10.1787/9789264097759-en

Denham A.M.J., Wynne O., Baker A.L., Spratt N.J., Turner A., Magin P. et al. (2019) "This is our life now. Our new normal": A qualitative study of the unmet needs of carers of stroke survivors. PLoS ONE, 14(5). DOI: https://doi.org/10.1371/journal.pone.0216682

Department of Health and Social Care, UK (2000). A National Strategy for Carers. URL: https://www.gov.uk/government/news/a-national-strategy-for-carers

Fevang E., Kverndokk S., Roed K. (2008). Informal care and labor supply. IZA Discussion Paper Series No. 3717. Bonn: IZA. URL: http://ftp.iza.org/dp3717.pdf

Gérain P., Zech E. (2019). Informal Caregiver Burnout? Development of a Theoretical Framework to Understand the Impact of Caregiving. Frontiers in Psychology, 10, 1748. DOI: https://doi.org/10.3389/fpsyg.2019.01748

Greenwood N., Pound C., Brearley S., Smith R. (2019) A qualitative study of older informal carers' experiences and perceptions of their caring role. Maturitas, 124, 1-7. DOI: https://doi.org/10.1016/j.maturitas.2019.03.006

Grundy E., Henretta J.C. (2006). Between elderly parents and adult children: a new look at the intergenerational care provided by the 'sandwich generation. Ageing and Society, 26, 707722. DOI: https://doi.org/10.1017/S0144686X06004934

Heitmueller A., Inglis K. (2004). Carefree? Participation and pay differentials for informal carers in Britain. IZA Discussion Paper Series No. 1273. Bonn: IZA. URL: http://ftp.iza.org/dp1273.pdf

Heitmueller, A. (2007). "The Chicken or the Egg? Endogeneity in Labour Market Participation of Informal Carers in England". Journal of Health Economics, 26(3).

DOI: https://doi.org/10.1016/j.jhealeco.2006.10.005

Henwood M. (1998). Ignored and Invisible? Carers Experience of the NHS. London: Carers' National Association.

Johnson R.W., Lo Sasso A.T. (2000). The Trade-Off between Hours of Paid Employment and Time Assistance to Elderly Parents at Mid-Life. Washington, DC: The Urban Institute. URL: https://www.urban.org/research/publication/trade-between-hours-paid-employment-andtime-assistance-elderly-parents-midlife/view/full_report

Katz S., Ford A.B., Moskowitz R.W., Jackson B.A., Jaffe M.W. (1963). Studies of Illness in the Aged: The Index of ADL: A Standardized Measure of Biological and Psychosocial Function. JAMA, 185 (12), 914-919. DOI: https://doi.org/10.1001/jama.1963.03060120024016

Lane P., McKenna H., Ryan A. (2003). Fleming P. The Experience of the Family Caregivers' Role: A Qualitative Study. Research and Theory for Nursing Practice, 17(2), 137-151(15). DOI: https://doi.org/10.1891/rtnp.17.2.137.53173 
Latif E. (2013). Labour supply effects of informal caregiving in Canada. Canadian Public Policy, 32, 413-429. DOI: https://doi.org/10.3138/Q533-8847-3785-1360

Li S., Song L., Feldman M.W. (2009). Intergenerational support and subjective health of older people in rural China: a gender-based longitudinal study. Australasian Journal of Ageing, 28(2), 81-86. DOI: https://doi.org/10.1111/j.1741-6612.2009.00364.x

McGarrigle Ch., Kenny R.A. (2013). Profile of the sandwich generation and intergenerational transfers in Ireland. The Irish Longitudinal Study on Ageing. Dublin: Trinity College Dublin.

Neundorfer M.M. (1991). Family Caregiving of the Frail Elderly: Impact of Caregiving on their Health and Implications for Interventions. Community Health, 14, 48-58. DOI: https://doi.org/10.1097/00003727-199107000-00007

Niimi Y. (2016). The "Costs" of Informal Care: An Analysis of the Impact of Elderly Care on Caregivers' Subjective Well-being in Japan. Review of Economics of the Household, 14, 779-810. DOI: https://doi.org/10.1007/s11150-016-9333-1

Rodrigues R., Schulmann K., Schmidt A., Kalavrezou N., Matsaganis M. (2013). The indirect costs of long-term care. Research note 8. European Commission. URL: https://ec.europa.eu/social/BlobServlet?docId=11780\&langId=en Rosstat [Federal State Statistics Service] (2018).

Rabochaya sila, zanyatost' i bezrabotitsa v Rossii - 2018 [Labor force, employment and unemployment in Russia - 2018] URL: https://gks.ru/bgd/regl/B18_61/Main.htm (data downloaded on 29.06.2020).

Rosstat [Federal State Statistics Service] (2008). Ekonomicheskaya aktivnost' naseleniya Rossii - 2008 [Economic activity of the population of Russia] URL: https://gks.ru/bgd/regl/B08_61/Main.htm (data downloaded on 29.06.2020).

Schulz K. (2000). Handbook on Dementia Caregiving, Evidence-Based. Interventions for Family Caregivers. New York: Springer.

Schulz R. et al. (1995). Psychiatric and Physical Morbidity Effects of Dementia Caregiving: Prevalence, Correlates, and Causes. Gerontolgist, 35, 771-791.

DOI: https://doi.org/10.1093/geront/35.6.771

Schulz R., Sherwood P. (2008). Physical and mental health effects of family caregiving. Journal of American Nursing, 108, 23-27.

DOI: https://doi.org/10.1097/01.NAJ.0000336406.45248.4c

Toseland R.W., Smith G., McCallion P. (2001). Family Caregivers of the Frail Elderly. Prepared for the US Administration on Aging. Washington, DC: US Department of Health and Human Services.

Van den Berg B., Fiebig D.G., Hall J. (2014). Well-being losses due to care-giving. Journal of Health Economics, 35, 123-131. DOI: https://doi.org/10.1016/j.jhealeco.2014.01.008

Van Houtven C.,Carmichael F., Jacobs J, Coyte P.C. (2020). The Economics of Informal Care. Oxford University Press USA.

Viitanen T.K. (2010). Informal eldercare across Europe: estimates from the European Community Household Panel. Economic Analysis and Policy, 40, 149-178.

DOI: https://doi.org/10.1016/S0313-5926(10)50023-7 